Deepika L. Chona, MD; Matthew R. Tubb, MD, PhD; Norman H. Gilinsky, MD, MRCP, FACG Division of Digestive Diseases, University of Cincinnati College of Medicine, $\mathrm{OH}$ (Drs. Chona and Gilinsky); Department of Family and Community Medicine, University of Cincinnati College of Medicine (Dr. Tubb)

Dnorman.gilinsky@uc.edu

The authors reported no potential conflict of interest relevant to this article.

doi: 10.12788/jfp.0266

\title{
Dyspepsia: A stepwise approach to evaluation and management
}

\author{
Let a patient's age and specific symptoms steer your \\ initial investigation. Consider treatment options beyond \\ antibiotics for $\mathrm{H}$ pylori gastritis and PPIs.
}

\section{PRACTICE RECOMMENDATIONS}

> Test for Helicobacter pylori in patients who are $<60$ years of age or who have no alarm symptoms. If results are negative, consider a trial of proton pump inhibitor therapy. (C)

\section{> Arrange for} esophagogastroduodenoscopy in individuals $\geq 60$ years of age and all patients with alarm symptoms, to identify or rule out a structural cause. (C)

> Consider a diagnosis offunctional dyspepsia if the work-up is negative. Supportive therapy, including the use of tricyclic antidepressants, prokinetics, and a holistic approach to lifestyle changes in select patients have shown encouraging results. (C)

Strength of recommendation (SOR)

A Good-quality patient-oriented evidence

B Inconsistent or limited-quality patient-oriented evidence

C Consensus, usual practice, opinion, disease-oriented evidence, case series
7 he global prevalence of dyspepsia is approximately $20 \%,{ }^{1}$ and it is often associated with other comorbidities and overlapping gastrointestinal complaints. The effects on the patient's quality of life, including societal impacts, are considerable. Symptoms and their response to treatment are highly variable, necessitating individualized management. While some patients' symptoms may be refractory to standard medical treatment initially, evidence suggests that the strategies summarized in our guidance here-including the use of tricyclic antidepressants (TCAs), prokinetics, and adjunctive therapies-may alleviate symptoms and improve patients' quality of life.

\section{What dyspepsia is-} and what it isn't

Dyspepsia is a poorly characterized disorder often associated with nausea, heartburn, early satiety, and bloating. The American College of Gastroenterology (ACG) now advocates using a clinically relevant definition of dyspepsia as "predominant epigastric pain lasting at least a month" as long as epigastric pain is the patient's primary complaint. ${ }^{2}$ Causes of dyspepsia are listed in TABLE 1.

Heartburn, a burning sensation in the chest, is not a dyspeptic symptom but the 2 may often coexist. In general, dyspepsia does not have a colicky or postural component. Symptoms that are relieved by evacuation of feces or gas generally should not be considered a part of dyspepsia.

I Functional dyspepsia (FD) is a subset for which no structural pathology has been identified, accounting for up to $70 \%$ of all patients with dyspepsia. ${ }^{3}$ The Rome Foundation, in its recent update (Rome IV), has highlighted 4 key symptoms and 2 proposed subtypes (TABLE 2 ). ${ }^{4}$ The comorbidities of anxiety, depression, and somatization appear to be more prevalent in these dyspepsia patients than in those with organic issues. The incidence of gastric malignancy is low in this cohort., 
TABLE 1

Causes of dyspepsia

\begin{tabular}{l|l}
\hline Common & Uncommon \\
\hline Alcohol gastritis & $\begin{array}{l}\text { Abdominal wall pain (including incisional hernia) } \\
\text { Food allergy } \\
\text { Biliary disease }\end{array}$ \\
$\begin{array}{l}\text { Food sensitivity } \\
\text { Functional }\end{array}$ & $\begin{array}{l}\text { Referred pain (musculoskeletal, diaphragmatic, thoracic) } \\
\text { Vascular causes (mesenteric ischemia, median arcuate }\end{array}$ \\
Helicobacter pylori gastritis & $\begin{array}{l}\text { ligament syndrome, mesenteric venous thrombosis, } \\
\text { aneurysms) }\end{array}$ \\
Hepatitis & \\
Large hiatal hernia & \\
Medications & \\
Pancreatic disorders & \\
Peptic ulcer disease &
\end{tabular}

a Nonsteroidal anti-inflammatory drugs, bisphosphonates, iron, corticosteroids, metformin, narcotics, antibiotics, and anticholinergics.

Dyspepsia occurring after an acute infection is referred to as postinfectious functional dyspepsia.

I Pathophysiology of functional dyspepsia. Dysmotility, visceral hypersensitivity, mucosal immune dysfunction, altered gut microbiota, and disturbed central nervous system processing contribute in varying degrees to the pathophysiology of FD. There is evidence that luminal factors have the potential to trigger local neuronal excitability. ${ }^{6,7}$ Early life psychosocial factors may further influence illness behaviors, coping strategies, stress responses, and the intensity of symptoms perceived by the patient. ${ }^{8}$

\section{Clues in the history and physical examination}

Patients describe their discomfort using a variety of terms, including pain, gnawing, burning, gassiness, or queasiness. Although allergic reactions to food (swelling of lips and tongue with a rash) are rare in adults, food intolerances are common in patients with dyspepsia. ${ }^{9}$ Consumption of nonsteroidal anti-inflammatory drugs is a common cause of dyspepsia, even at over-the-counter strength, and may cause ulceration, gastrointestinal bleeding, and anemia. Narcotic and marijuana use and the anticholinergic effects of antidepressant medications are associated with gastrointestinal dysmotility, including gastroparesis.

Patients with FD often exhibit symptoms of other functional abdominal disorders including irritable bowel syndrome, functional heartburn, bloating, or chronic nausea, and may have been previously diagnosed with overlapping conditions suggestive of visceral hypersensitivity, including depression, anxiety, fibromyalgia, migraine, and pelvic pain. During the patient's office visit, be alert to any indication of an underlying psychological issue.

I The initial diagnostic challenge is to identify those patients who may have a structural disorder requiring expedited and targeted investigation. Weight loss, night waking, and vomiting are unusual in the setting of either FD or Helicobacter pylori gastritis. These and other features of concern (TABLE 3) make a diagnosis of a functional disorder less likely and should prompt immediate consideration of abdominal imaging or endoscopic examination. Epigastric tenderness on palpation is common in patients with FD and is not necessarily predictive of structural pathology-unless accompanied by other findings of concern. Abdominal scars or a history of trauma may be suggestive of abdominal wall pain. Abdominal pain that remains unchanged or increases when the muscles of the abdominal
Weight loss, night waking, and vomiting make functional dyspepsia less likely and deserve immediate consideration of abdominal imaging or endoscopic examination. 
TABLE 2

Functional dyspepsia: Dx criteria and subtypes ${ }^{4}$

Presence of at least 1 of the following 4 symptoms ${ }^{\mathrm{a}, \mathrm{b}}$

Postprandial distress syndrome (symptoms at least $3 \mathrm{~d} / \mathrm{wk}^{\mathrm{c}}$ )

1. Postprandial fullness, and/or

2. Early satiation

Epigastric pain syndrome (symptoms at least $1 \mathrm{~d} / \mathrm{wk}^{\mathrm{c}}$ )

3. Epigastric pain, and/or

4. Epigastric burning

a Criteria fulfilled for the last 3 months, with symptom onset at least 6 months before diagnosis.

${ }^{b}$ No evidence of organic, systemic, or metabolic

disease likely to explain the symptoms on

routine investigations (including upper endoscopy).

'Severe enough to interfere with daily activities.

wall are tensed (Carnett sign) suggests abdominal wall pain.

\section{Initial testing and $\mathrm{Tx}$ assessments focus on $\mathrm{H}$ pylori}

All 3 of the major US gastroenterology organizations recommend a stepwise approach in patients without alarm symptoms, generally beginning (in those $<60$ years) by testing for $H$ pylori with either the stool antigen or urea breath test (UBT) - and initiating appropriate treatment if results are positive. ${ }^{5,10}$ (The first step for those $\geq 60$ years is discussed later.) Since the serum antibody test cannot differentiate between active and past infection, it is not recommended if other options are available. ${ }^{11}$ The stool antigen test is preferred; it is a costeffective option used for both diagnosis and confirmation of $H$ pylori eradication.

The UBT identifies active infection with a sensitivity and specificity of $>95 \%{ }^{12}$ but is more labor intensive, employs an isotope, and is relatively expensive. Because proton pump inhibitors (PPIs), bismuth, and antibiotics may increase the false-negative rate for both the UBT and stool antigen test, we recommend that these medications be held for 2 to 4 weeks prior to testing. ${ }^{11} \mathrm{H}_{2}$-receptor antagonists do not need to be restricted.

I Treatment regimens containing clarithromycin have fallen into disfavor given the high rates of resistance that are now encountered. Fourteen-day regimens that can be used empirically (without susceptibility testing) are bismuth quadruple therapy (bismuth, metronidazole, tetracycline, and PPI) or rifabutin triple therapy (rifabutin, amoxicillin, and PPI). ${ }^{13}$ To confirm eradication, perform repeat testing with either stool antigen or UBT no sooner than 4 weeks after completion of therapy. If the first treatment fails, try a second regimen using different antibiotics. ${ }^{14}$ Although the impact of $H$ pylori eradication on dyspeptic symptoms is only modest, this strategy is recommended also to reduce the risk of peptic ulceration and gastric neoplasia.

\section{Next-step testing and Tx considerations}

Given the heterogeneity of presenting symptoms of dyspepsia, some clinicians may be hesitant to diagnose a functional disorder at the first visit, preferring instead to conduct a limited range of investigations in concert with initial medical management. In these circumstances it would be reasonable, in addition to testing for $\mathrm{H}$ pylori, to order a complete blood count (CBC) and to measure serum lipase and liver enzymes. Keep in mind that liver enzymes may not be elevated in uncomplicated biliary colic.

Consider ultrasound imaging if gallstones are a consideration. A computerized tomography scan may not exclude uncomplicated and noncalcified gallstones, but it is an excellent modality for detecting suspected retroperitoneal pathology. Consider working with a gastroenterologist if the patient exhibits alarm features.

I Empiric PPI therapy. A trial of daily PPI use over 4 weeks is recommended for patients without $H$ pylori and for those whose symptoms continue despite eradication of the bacterium. A Cochrane meta-analysis found that PPI therapy was more effective than placebo ( $31 \%$ vs $26 \%$; risk ratio, 0.88 ; number needed to treat $[\mathrm{NNT}]=11 ; 95 \% \mathrm{CI}$ 0.82 to $0.94 ; P<.001) .{ }^{15} \mathrm{PPI}$ therapy appears to be slightly more effective than treatment with $\mathrm{H}_{2}$-receptor antagonists. Both are proposed in the United Kingdom guideline. ${ }^{16}$ Both are generally safe and well tolerated but are not 
TABLE 3

\section{Alarm features in dyspepsia ${ }^{a}$}

Age $\geq 60$ years $^{\mathrm{b}}$
Unintended weight loss
Dysphagia or odynophagia
Anemia
Gastrointestinal bleeding
Palpable mass or lymphadenopathy
Symptoms that have a recent onset and
progressive nature
Persistent vomiting
Family history of upper gastrointestinal cancer
Unsatisfactory response to empiric therapies if
no prior investigation
a Dyspepsia patients with these features or medical
histories require investigation for another disorder.
b Variable between 50 and 60 years depending on the
specific guideline employed.

without potential adverse effects when used long term.

Dietary modification. Patients with dyspepsia commonly report that meals exacerbate symptoms. This is likely due to a combination of gastric distension and underlying visceral hypersensitivity rather than food composition.

There is no reliable "dyspepsia diet," although a systematic review implicated wheat and high-fat foods as the 2 most common contributors to symptom onset. ${ }^{17}$ Recommended dietary modifications would be to consume smaller, more frequent meals and to eliminate recognized trigger foods. Patients with postprandial distress syndrome, a subset of FD, may want to consider reducing fat intake to help alleviate discomfort. If symptoms continue, evaluate for lactose intolerance. Also, consider a trial of a gluten-free diet. The lowFODMAP diet (restricting fermentable oligo-, di- and monosaccharides, as well as polyols) has shown benefit in patients with irritable bowel syndrome and may be considered in those with intractable FD, given the overlap in physiology of the disorders.

I Upper gastrointestinal endoscopy. The ACG has suggested that esophagogastroduodenoscopy (EGD) be performed as the first investigative step for patients $\geq 60$ years, while testing for $H$ pylori be considered as the first step in younger patients, even if alarm symptoms are present ${ }^{2}$ (FIGURE). This decision must be individualized, particularly in patients of Asian, Central or South American, or Caribbean descent, in whom the incidence of gastric cancer is higher with earlier onset. $^{18}$

Also consider EGD for patients whose symptoms have not improved despite eradication of $H$ pylori or an adequate trial of PPI therapy. While some guidelines do not require EGD in low-risk patients at this stage, other authorities would consider this step prudent, particularly when quality of life has been significantly impaired. An underlying organic cause, mainly erosive esophagitis or peptic ulcer disease, is found in $20 \%$ to $30 \%$ of patients with dyspepsia. ${ }^{5}$

Most patients without alarm features, with normal findings on upper endoscopy, who do not have $H$ pylori gastritis, and whose symptoms continue despite a trial of PPI therapy, will have FD (FIGURE). ${ }^{2}$

\section{Offer patients with functional dyspepsia supportive therapy Neuromodulators}

I TCAs are superior to placebo in reducing dyspeptic symptoms with an NNT of 6 and are recommended for patients with ongoing symptoms despite PPI therapy or $H$ pylori eradication. ${ }^{2}$ Begin with a low dose and increase as tolerated. It may take a few weeks for improvement to be seen. Exercise caution in the presence of cardiac arrhythmias.

I Mirtazapine, 7.5 to $15 \mathrm{mg}$ every night at bedtime, reduces fullness and bloating in postprandial distress syndrome and is useful for patients who have lost weight. It's important to note that TCAs and mirtazapine both have the potential for QT prolongation, as well as depression and suicidality in younger patients. ${ }^{19}$ The anxiolytic buspirone, $10 \mathrm{mg}$ before meals, augments fundic relaxation, improves overall symptom severity, and helps alleviate early satiety, postprandial fullness, and upper abdominal bloating. ${ }^{20}$

\section{Prokinetics}

A recent meta-analysis demonstrated significant benefit in symptom control in dys-
The stool antigen test is preferred for diagnosing and confirming eradication of $H$ pylori. The serum antibody test cannot differentiate between active and past infection. 


\section{Suggested work-up for a patient with dyspepsia}

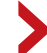

Because PPIs, bismuth, and antibiotics may increase the false-negative rate for both the UBT and stool antigen test, we recommend that these medications be held for 2 to 4 weeks prior to testing.

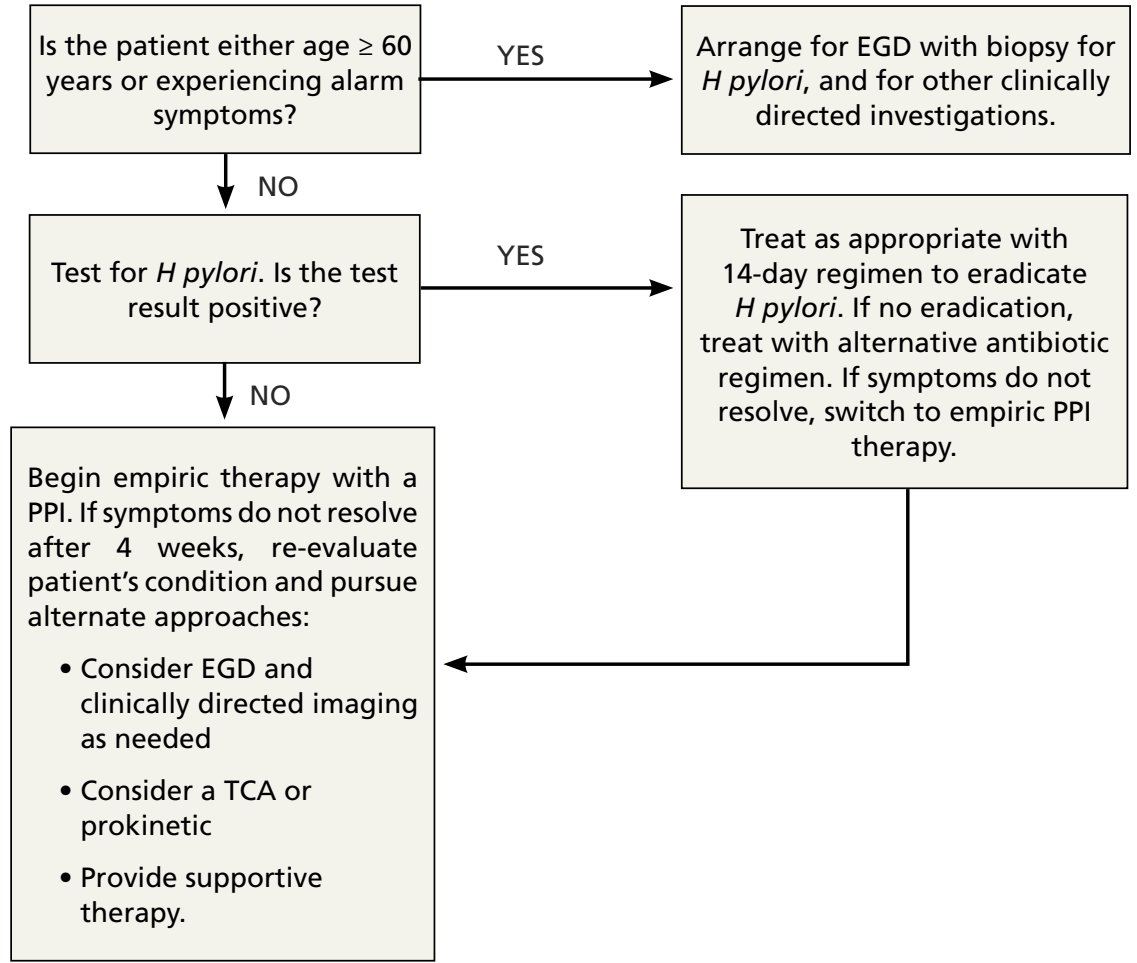

EGD, esophagogastroduodenoscopy; H pylori, Helicobacter pylori; PPI, proton pump inhibitor; TCA, tricyclic antidepressant.

peptic patients treated with prokinetics $(\mathrm{NNT}=7){ }^{21}$ However, the benefit was predominantly due to cisapride, a drug that was withdrawn from the US market due to adverse effects. There are no clinical trials of metoclopramide or domperidone (not available in the United States) in FD. Nonetheless, the ACG has given a conditional recommendation, based on low-quality evidence, for the use of prokinetics in patients with FD not responding to PPI therapy, H pylori eradication, or TCA therapy. ${ }^{2}$

I A shortcoming of the established guidelines is that they do not provide guidance as to long-term management of those patients who respond to prescription medications. Our practice has been to continue medications for a minimum of 3 months, then begin a slow taper in order to establish the lowest efficacious dose. Some patients may relapse and require full dosage for a longer period of time.

\section{Adjunctive therapies} are worth considering I Complementary and alternative medicines. Products containing ginger, carraway oil, artichoke leaf extract, turmeric, and red pepper are readily available without prescription and have long been used with variable results for dyspepsia. ${ }^{22}$ The 9-herb combination STW-5 has demonstrated superiority over placebo in a number of studies and has a favorable safety profile. ${ }^{23}$ The recommended dose is 10 to 20 drops tid. The European manufacturer has recently modified the package insert noting rare cases of hepatotoxicity. ${ }^{24}$

A commercially available formulation (FDgard) containing L-menthol (a key component of peppermint oil) and caraway has been found to reduce the intensity of symptoms in patients with FD. Potential adverse effects include nausea, contact dermatitis, bronchospasm, and atrial fibrillation. Cayenne, a red 
pepper extract, is available over the counter for the benefit for epigastric pain and bloating. Begin with a 500-mg dose before breakfast and a 1000-mg dose before dinner, increasing to $2500 \mathrm{mg} / \mathrm{d}$ as tolerated. Cayenne preparations may trigger drug toxicities and are best avoided in patients taking antihypertensives, theophylline, or anticoagulants.

Cognitive behavioral therapy, acupuncture, and hypnosis. These modalities are time consuming, are often expensive, are not always covered by insurance, and require significant motivation. A systematic review found no benefit. ${ }^{25}$ Subsequent studies summarized in the ACG guidelines ${ }^{2}$ reported benefit; however, a lack of blinding and significant heterogeneity among the groups detract from the quality of the data. It remains unclear whether these are effective strategies for FD, and therefore they cannot be recommended on a routine basis.

\section{CORRESPONDENCE}

Norman H. Gilinsky, MD, Division of Digestive Diseases, University of Cincinnati College of Medicine, 231 Albert Sabin Way, Cincinnati, OH 45267-0595; norman.gilinsky@ uc.edu

\section{References}

1. Ford AC, Marwaha A, Sood R, et al. Global prevalence of, and risk factors for, uninvestigated dyspepsia: a meta-analysis. Gut. 2015;64:1049-1057.

2. Moayyedi P, Lacy BE, Andrews CN, et al. ACG and CAG clinical guideline: management of dyspepsia. Am J Gastroenterol. 2017;112:988-1013.

3. Ford AC, Marwaha A, Lim A, et al. What is the prevalence of clinically significant endoscopic findings in subjects with dyspepsia? Systematic review and meta-analysis. Clin Gastroenterol Hepatol. 2010;8:830-837.

4. Stanghellini V, Chan FKL, Hasler WL, et al. Gastroduodenal disorders. Gastroenterology. 2016;150:1380-1392.

5. Shaukat A, Wang A, Acosta RD, et al. The role of endoscopy in dyspepsia. Gastrointest Endosc. 2015;82:227-232.

6. Wauters L, Talley NJ, Walker MM, et al. Novel concepts in the pathophysiology and treatment of functional dyspepsia. Gut 2020;69:591-600

7. Weinstock LB, Pace LA, Rezaie A, et al. Mast cell activation syndrome: a primer for the gastroenterologist. Dig Dis Sci. 2021;66:965-982.

8. Drossman DA. Functional gastrointestinal disorders. History, pathophysiology, clinical features and Rome IV. 2016. Accessed August 16, 2021. www.gastrojournal.org/article/S0016-5085 (16)00223-7/fulltext

9. Boettcher E, Crowe SE. Dietary proteins and functional gastrointestinal disorders. Am J Gastroenterol. 2013;108:728-736.

10. Talley NJ, AGA. American Gastroenterological Association medical position statement: evaluation of dyspepsia. Gastroenterol. 2005;129:1753-1755.

11. El-Serag HB, Kao JY, Kanwal F, et al. Houston Consensus Conference on testing for Helicobacter pylori infection in the United States. Clin Gastroenterol Hepatol. 2018;16:992-1002.

12. Ferwana M, Abdulmajeed I, Alhajiahmed A, et al. Accuracy of urea breath test in Helicobacter pylori infection: meta-analysis. World J Gastroenterol. 2015;21:1305-1314.

13. Howden CW, Graham DY. Recent developments pertaining to H. pylori infection. Am J Gastroenterol. 2021;116:1-3.

14. Chey WD, Leontiadis G, Howden W, et al. ACG clinical guideline: treatment of Helicobacter pylori infection. Am J Gastroenterol. 2017;112:212-239.

15. Pinto-Sanchez MI, Yuan Y, Hassan A, et al. Proton pump inhibitors for functional dyspepsia. Cochrane Database Syst Rev. 2017;11:CD011194.

16. National Institute for Health and Care Excellence. Gastrooesophageal reflux disease and dyspepsia in adults: investigation and management. [Clinical guideline] Accessed August 6, 2021 www.ncbi.nlm.nih.gov/books/NBK552570/

17. Duncanson KR, Talley NJ, Walker MM, et al. Food and functiona dyspepsia: a systematic review. J Hum Nutr Diet. 2018;31:390-407.

18. Lin JT. Screening of gastric cancer: who, when, and how. Clin Gastroenterol Hepatol. 2014;12:135-138.

19. Spielmans GI, Spence-Sing T, Parry P. Duty to warn: antidepressant black box suicidality warning is empirically justified. Front Psychiatry. 2020;11:1-18.

20. Tack J, Janssen P, Masaoka T, et al. Efficacy of buspirone, a fundusrelaxing drug in patients with functional dyspepsia. Clin Gastroenterol Hepatol. 2012;10:1239-1245.

21. Pittayanon R, Yuan Y, Bollegala NP, et al. Prokinetics for functional dyspepsia: a systemic review and meta-analysis of randomized controlled trials. Am J Gastroenterol. 2019;114:233-243.

22. Deutsch JK, Levitt J, Hass DJ. Complementary and alternative medicine for functional gastrointestinal disorders. Am J Gastroenterol. 2020;115:350-364.

23. Malfertheiner P. STW 5 (iberogast) therapy in gastrointestinal functional disorders. Dig Dis. 2017;35:25-29.

24. Sáez-González E, Conde I, Díaz-Jaime FC, et al. Iberogastinduced severe hepatotoxicity leading to liver transplantation. Am J Gastroenterol. 2016;111:1364-1365.

25. Soo S, Forman D, Delaney B, et al. A systematic review of psychological therapies for nonulcer dyspepsia. Am J Gastroenterol. 2004;99:1817-1822.
FIND YOUR NEXT JOB AT

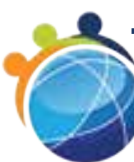
MEDJOBNETWORK, com Physician $\odot$ NP/PA Career Center

\section{The first mobile job board for Physicians,} NPs, and PAs

Mobile Job Searches-access MedJobNetwork.com on the go from your smartphone or tablet

Advanced Search Capabilities - search for jobs by specialty, job title, geographic location, employers, and more

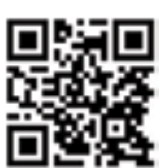

Scan this QR code to access the mobile version of MedJobNetwork.com 